\title{
The service life increasing of agricultural machinery products by electro-acoustic spraying
}

\author{
Andrei Kochetov ${ }^{1}$,Elena Fisunova ${ }^{1}$, Tatyana Lavrenova ${ }^{1, *}$, Olga Baryshnikova $^{1}$, and \\ Nadezhda Metelkova ${ }^{1}$ \\ ${ }^{1}$ Don State Technical University, 1, Gagarin square, 344003, Rostov-on-Don, Russia
}

\begin{abstract}
This publication is devoted to the formation of equilibrium conditions in ordered dislocation structures obtained by the influence of highly concentrated electric energy flows and an informal (longitudinaltorsional) ultrasonic field on the conductive substrate material.
\end{abstract}

\section{Introduction}

The problem the service life increasing for machine parts and forming tools is most acute for both domestic and foreign manufacturers of engineering products. The solution of this problem is inherently connected with the use of modern strengthening technologies based on the use of highly concentrated energy sources that allow to obtain ordering in dislocation structures of materials.

One of the most promising directions for the formation of ordered dislocation structures is the usage of a complex source of a powerful ultrasonic field in the electroacoustic spraying process. This method of wear-resistant protective coatings obtaining is based on the complex interaction of highly concentrated sources of electrical energy and a longitudinal torsion ultrasonic field, and physical and chemical processes and phenomena occurring during electroacoustic spraying are quite complex and ambiguous, and require more detailed and in-depth research.

Obtaining of ordered dislocation structures under the influence of a powerful longitudinal-torsional ultrasonic field and an electric spark in metals can stimulate the development of a number of scientific and applied works in this field.

\section{The influence mechanism of solid surface films on deformation and destruction}

Since 1934, when Rosky found that an oxide film with a thickness of less than 20 atomic layers increases the critical shear stress of cadmium single crystals by more than $50 \%$, the question of the influence of solid surface films on the behavior of crystals under load has

\footnotetext{
*Corresponding author: bys_ka87@mail.ru
} 
been constantly under the attention of scientists.

Numerous experiments conducted with the usage of various base crystals and surface films (both oxide [1,2] and metal [3]) confirmed in most cases the effect having been found by Rosky. It also turned out that surface films significantly reduce the creep rate of single crystals [4]. It is characteristic that the influence of the surface film appears already at the earliest stages of deformation.

The displacement of the stress - strain curve towards higher stresses cannot be explained based on the assumption of a simple load distribution between the base crystal and the film, since the calculated film strength required for such flow stress increasing is incredibly large. Therefore, the presence of a surface film leads to the mechanical property change of the base crystal. In our opinion, there are three possible mechanisms of influence that do not contradict the research results by other scientists:

- alloy formation in the surface layer of the base crystal, which causes hardening of this layer;

- fixing surface sources of Frank-Reid;

- dislocation reaching preventing on the surface of the base crystal, i.e., sliding suppressing.

Gilman's spectrographic analysis of zinc crystals reinforced with copper surface films did not reveal any significant doping of the surface layer. X-ray analysis of pure and copper-coated zinc crystals subjected to deformation showed that the distortion of the surface layer in the base crystal is greater in the case of coated samples [5]. This result is easy to interpret if we assume that the film is a barrier that prevents dislocations reaching on the surface. Indeed, if the mechanism of film influence consisted in action suppressing of dislocation surface sources, the distortion should be less for the coated crystal. Large distortions on the surface layer of the coated crystal are caused by groups of dislocations accumulated under the film. The conclusion about the "barrier" mechanism of surface film influence on metal deformation is confirmed by the study results of internal friction in zinc samples coated with an oxide film [6]. The latter also cannot be explained on the basis of the assumption of surface sources fixing.

Thus, the degree of film influence on metal deformation is determined by its ability to prevent dislocation reaching on the surface. The factors that determine the effectiveness of the film as a barrier to dislocations are discussed below.

\section{Interaction of a single dislocation with a film surface}

Phase boundaries with different elastic properties have a significant effect on the dislocations contained in the solid. This effect is due to the fact that certain boundary conditions must be respected on the phase interface surfaces. Satisfaction of these conditions can significantly change the nature of elastic deformations created by dislocations in comparison with the case of an infinite homogeneous body. As a result, forces may appear that seek to change the dislocation behavior in such a way to change the elastic energy of the body.

The interaction model of a single dislocation with a surface film can be represented as follows. Consider the case of a " thick "(semi-infinite) "film". We assume that the base crystal has a shift $\mathrm{G}_{1}$ modulus and is located to the right of the phase interface $(0<x<$ $\infty)$. The "Film" with the shift $\mathrm{G}_{2}$ modulus is located to the left of the interface $(-\infty<x<$ 0 ). In the base crystal at a distance $l$ from the boundary (Fig.1), there is an infinite helical dislocation with power $b^{2}$ (where $\mathrm{b}$ - is the vector by Burgers), parallel to boundary"film-crystal". 
To determine the force acting on the dislocation from the "film" side, we can use the Eshelby analogy between the stress field of a screw dislocation in a medium with variable elastic characteristics and a similar problem of electrostatics for a linear charge in a medium with a non-uniform dielectric constant. If the band over the interface is perfect, then the displacements and stresses are continuous at the boundary (i.e. at $\mathrm{x}=0$ ). It is easy to show [7] that these conditions will be carried out if the "image" dislocation is placed at point $(-l, 0)$ with power $\mathrm{Kb}^{2}$ where $K=\frac{\left(G_{2}-G_{1}\right)}{\left(G_{2}+G_{1}\right)}$. Force acting on dislocation from the border "film-crystal" (Fig. 1)

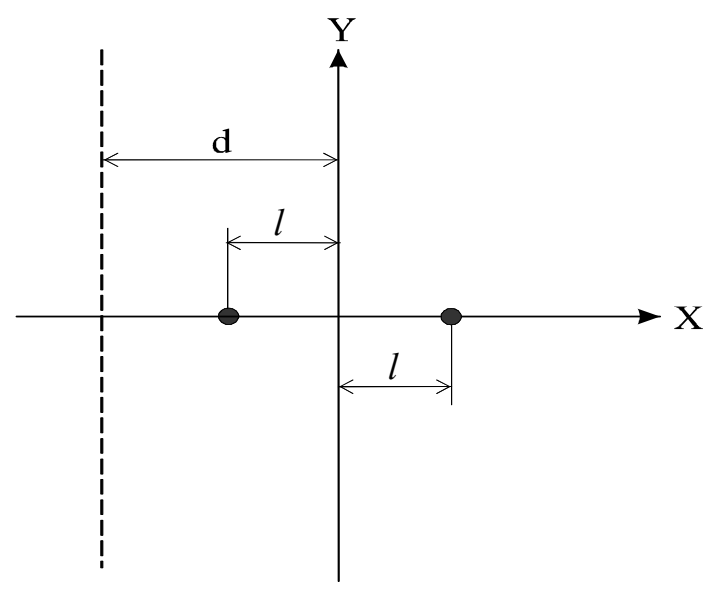

Fig. 1. Single dislocation near the interface is equal to the interaction force of this dislocation with the "image" dislocation, i.e.

$$
F=\frac{G_{1} K b^{2}}{4 l \pi}
$$

If $G_{2}>G_{1}(K>0)$, the dislocation is repelled from the border, and if $G_{2}>G_{1}(K<$ $0)$, it is attracted. Thus, a dislocation located in the base crystal is attracted to the boundary if the film is softer than the base crystal, and repels in the opposite case. If the crystal borders on a vacuum $\left(G_{2}=0, K=-1\right)$, then the dislocation is affected by of attraction force $F=\frac{G_{1} K b^{2}}{4 l \pi}$, seeking to bring dislocation to the surface.

Let's now consider the case when the film thickness is finite and equal $\mathrm{d}$ (marked with a dotted line in Fig. 1). To satisfy the boundary conditions, it is necessary to introduce an infinite series of "image" dislocations [7], which are located at points: $-l,-(l+2 d),-(l+$ $4 d),-(l+6 d)$, and so on, and their power is equal to, respectively: $K,-\left(1-K^{2}\right),-(1-$ $\left.K^{2}\right) \cdot K,-\left(1-K^{2}\right) \cdot K^{2}, \ldots$ To determine the interaction force of the dislocation with the film, we find the total sum of forces acting on the real dislocation from an infinite number of "image" dislocations

$$
F_{1}=\frac{G_{1} K b^{2}}{4 l \pi} \cdot\left\{1-\left(1-K^{2}\right) \cdot \sum_{n-1}^{\infty} K^{n-1} \frac{\alpha}{\alpha+n}\right\}
$$

where $\alpha=l / d$.

Analysis of formula (2) shows that if the film is softer than the crystal $(K<0)$, the dislocation is always attracted to the boundary. In the opposite case $(K>0)$, the picture becomes more complex. At large distances $(l \ll d, \alpha \ll 1)$, the dislocation is attracted to the film, and at small distances $(l \ll d, \alpha \ll 1)$, it is repelled from it. Therefore, at a 
certain distance $l_{0}$, the force of the "image" is zero, i.e. the dislocation is in a state of stable equilibrium, which can be determined by equating the expression (2) to zero.

Formulas that accurately describe the interaction of the edge dislocation with the phase boundaries are much more complex than for the helical dislocation $[8,9]$. However, the force of interaction of the edge dislocation with the boundary in the case of a "thick film" can be approximately expressed by the formula (1). the Maximum error does not exceed $15 \%$ [7]. The exact solution for the case of a film of finite thickness $[9,10]$ allows us to determine the equilibrium position of the edge dislocation, which is closer to the boundary than for the screw dislocation. This difference increases with the ratio $G_{2} / G_{1}$.

The above applies to a situation where the crystal-base does not interact with the film. There are two other possibilities:

a) at the "film-crystal" boundary, a compound is formed with a shear modulus $G_{3}$;

b) the film and base materials form a series of solid solutions, and the shear modulus of the intermediate layer changes continuously from $G_{1}$ to $G_{2}$.

\section{Simplified analytical model of interaction of a single dislocation with the resulted layers by electroacoustic spraying}

Currently in many cases researchers have achieved much more success in the development of acceptable hardening technologies than in the physical justification of the methods and processes themselves, as well as methods for predicting for the strengthening coatings and their properties, depending on the conditions of subsequent operation.

This situation often leads to unjustified ignoring and sometimes not understanding the need for deep physical research of hardening processes and related phenomena from the perspective of fundamental sciences. On the other hand, the advance in the development of applied issues is due not only to obvious utilitarian and pragmatic considerations, but also to the fact that to a certain extent this is due to the objective difficulties of general physical approaches using and model representations in relation to the phenomena accompanying the processes of hardening, friction and wear. The mathematical apparatus(model) is poorly developed, i.e. researchers are forced to solve the problems of hardening at the engineering level.

The lack of reliable mathematical models of hardening processes that take into account the stochastic nature of many related phenomena makes it impossible to objectively assess the effectiveness of various hardening technologies.

The double barrier created by electroacoustic spraying prevents reaching dislocations on the surface. The conclusion about the barrier mechanism of influence is confirmed by numerous experiments conducted using various base crystals and applied surface layers (both oxide [1, 2] and metal [3, 11]). in most cases, the found effect by Rossky was confirmed. Based on the previously described material, a simplified analytical model of the interaction of a single dislocation with the resulting layers by electroacoustic deposition is proposed (Fig. 3).

Let us now analyze the case when a compound with a shear modulus is formed at the film - metal boundary $G_{3}$. If we limit ourselves in the case of a "thick layer", then the force acting on the dislocation in the metal from the side of the intermediate layer of thickness $d$, which has a shear modulus equal to $G_{3}$

$$
F_{2}=\frac{G_{1} b^{2} K_{31}}{4 l \pi} \cdot\left\{1-\left(1-K_{31}^{2}\right) \cdot \sum_{n-1}^{\infty}\left(-K_{23}\right)^{n} K_{31}^{n+2} \frac{\alpha}{\alpha+n}\right\},
$$

where $K_{i j}=\left(G_{i}-G_{j}\right) /\left(G_{i}+G_{j}\right)$. 


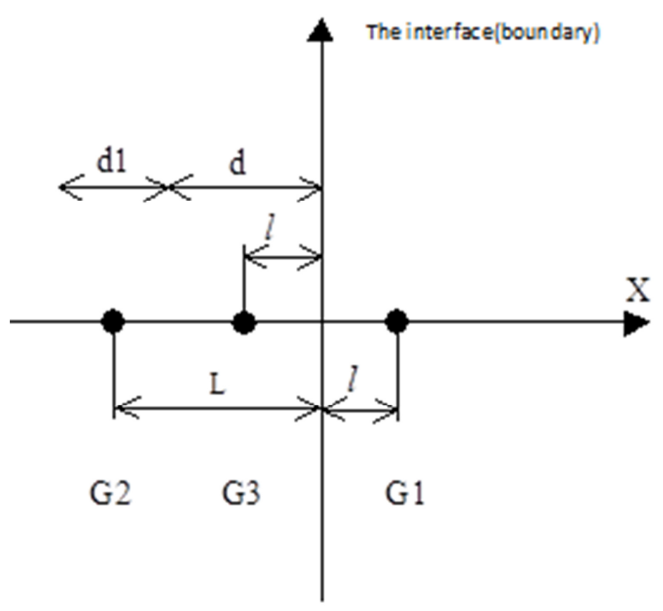

Fig. 2. A single dislocation near the interface(boundary) "coated metal layer ": $G_{1}$ - the shear modulus of the material of the hardened sample; $G_{2}$ - the shear modulus of the deposited layer; $G_{3}$ - the shear modulus of plastically deformed layer; $d$ - the thickness of plastically deformed layer; $d l$ - the thickness of the sprayed layer; $l$ - distance from the dislocation to the interface; $L$ - distance from the dislocation of the image of the sprayed layer to the interface.

Depending on the ratio of shift modules, the "image" force has a different character [12].

Using the superposition principle, we can say that the resulting force acting from the obtained layers on the dislocation in the metal is defined as the sum of forces applied to it

$$
\bar{F}_{p}=k \bar{F}_{1}+\bar{F}_{2}
$$

where $\mathrm{k} \approx l / L$.

\section{Equilibrium conditions for ordered dislocation structures}

Before determining how to relax the stresses associated with such a blocked series, it is necessary to study the distribution of these stresses (and, consequently, the distribution of dislocations in the series) depending on the thickness and stiffness of the film obtained by electroacoustic spraying.

Let's assume that there are $n$ parallel screw dislocations in the base crystal, located on the axis $x$ at points $x_{1}, x_{2} \ldots, x_{n}$. A tangent stress $\tau$ is applied to the crystal, under the action of which the dislocations move to the phase interface. Let us first turn to the case of a semiinfinite film. Each of $n$ dislocations interacts with the other $(n-1)$ dislocations of the "image". In a state of static equilibrium, the force acting on the dislocation in the cluster from all other dislocations must balance the force caused by the applied stress [1]. Based on the above material, we obtain a system of equations for determining the positions of dislocation equilibrium

$$
\underset{2 \pi}{\mathrm{G}_{1} \mathrm{~b}}\left\{\sum_{\substack{i=1 \\ j \neq i}}^{n} \frac{1}{x_{j}+x_{i}}+K \sum_{i=1} \underset{x_{j}+x_{i}}{-1}\right\}=\tau, j=1,2, \ldots, n
$$

where $G_{1}$ is the shear modulus of the substrate; $K=\left(G_{2}-G_{1}\right) /\left(G_{2}+G_{1}\right) ; G_{2}$ - the shear modulus of the film; $b$ - the module of Burgers vector; $\tau$ - voltage. 
The first system of equations represents the stress acting on a dislocation located at a point $x_{j}$, from the side of $n-1$ real dislocations located at points $x_{i}$ the second - the stress caused by a number $n$ of dislocations of the image power $K b^{2}$ placed at points $-x_{j}$. With the increase in rigidity of the film the equilibrium position of dislocations is shifted further from the phase boundary.

Numerical calculations are difficult for large values $n$. However, in this case, we can use the approximation of continuously distributed dislocations [2]. The equilibrium condition is written as follows

$$
\int \frac{f(t) d t}{x-t}+K \int_{0}^{L} \frac{f(t) d t}{x+t}=\frac{2 \pi \tau}{G_{1} b}, 0 \leq x \leq L,
$$

where $L$ is the length of the cluster (accumulation); $f(t)$ - unknown dislocation distribution function.

The first integral is defined here in the sense of the main Cauchy value. Solving the integral equation (6), we find the distribution function $f(t)$, the value of which allows us to determine the number of dislocations in the cluster and the stress field caused by this cluster.

The presence of a film in front of the slip band changes the number and distribution of dislocations in the cluster. For the case $0 \leq K \leq 1$ the relationship between the value $L / n$ and $K$ can be represented with very good accuracy by a linear relation

$$
\left(\frac{2 \pi \tau}{G_{1} b}\right) \cdot \frac{L}{n} \approx 2+(\pi-2) \cdot K
$$

Fig. 1 shows the dependence of tangent stress on the sliding plane $y=0$ in the film on the distance to the boundary for various object $K$ (the head dislocation lies on the boundary). The homogeneous medium corresponds to a value $K=0$ that decreases with increasing film stiffness $\tau_{Y Z}$.

For the case of a film of finite thickness $d$, the dislocation equilibrium condition is written as follows

$$
\frac{G_{1} b}{2 \pi}\left\{\sum_{\substack{i=1 \\ i \neq j}}^{n} \frac{1}{x_{j}+x_{i}}+K\left[\sum_{i=1}^{n} \frac{1}{x_{j}+x_{i}}-\sum_{v=1}^{\infty} \frac{\left(1-K^{2}\right) \cdot K^{v-2}}{x_{j}+x_{i}+2 v d}\right]\right\}=\tau,
$$

where $j=1,2, \ldots, n ; ; v$ - is the Poisson's ratio.

\section{Results of experimental studies}

The study of chemical element distribution across the cross-section of the hardened layer, performed by electron microscopy on the scanning electron microscope "Kamebaks-micro" using a microprobe, secondary electrons and an absorbing current, showed a high heterogeneity of their distribution across the cross-section of the layer. The research is related to the study of the nature of element distribution in a layer reinforced sequentially with anodes made of various materials [13-14]. For an example, in Fig.3 the distribution of elements in the hardened layer is shown, which has been obtained on a matrix of steel 20 in series by anodes of VK8 and copper.

Qualitative analysis of the distribution shows that all the elements included in the layer are distributed differently in thickness. So, iron is present on the surface of the layer (slightly) and on the border of the layer with the matrix; cobalt is distributed throughout the whole layer, and in the middle we can see its maximum amount; copper is distributed in small quantities in the near-surface layer. 


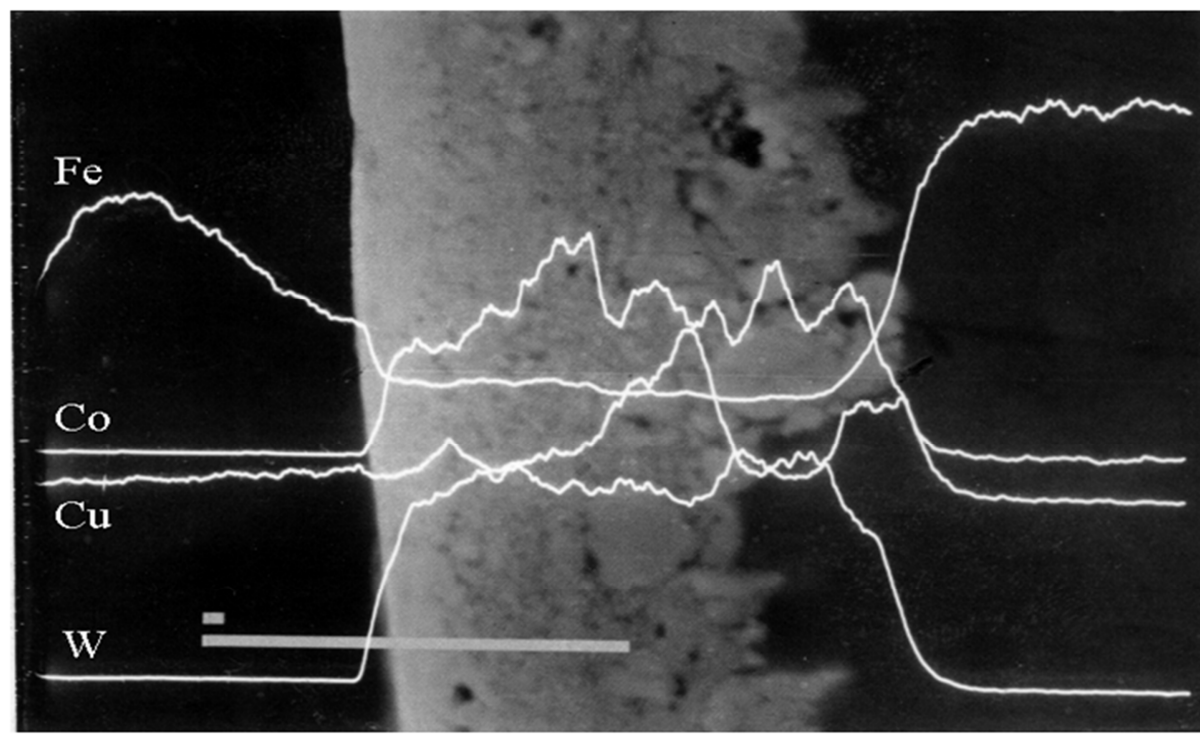

Fig. 3. Distribution of the element composition in the sprayed layer (marker mark corresponds to 10 microns).

\section{Conclusions}

Based on theoretical and experimental studies of the effect of surface films on the ordering of dislocation structures obtained by electroacoustic spraying, the following conclusions can be drawn:

It is found that for a certain ratio of film-crystal $G_{2} / G_{1}$ shift modules, the number of edge dislocations in the cluster (accumulation) decreases with increasing Poisson's ratio.

The higher the film modulus (i.e., the larger $K$ is), the smaller the number of dislocations in the cluster at the same applied voltage we can see. Thus, a crystal covered with a rigid film will be plastically deformed less than a crystal without a film under the same stress (provided that the average path length of dislocations in the crystal is not very small).

The most acceptable explanation for crystal hardening is surface films, which prevent reaching dislocations on the surface by sliding suppressing and ordering dislocation structures.

The process of electroacoustic deposition allows to obtain a "double barrier" that prevents from reaching dislocations the surface. The first barrier is caused by microplastic deformation of the surface layer of the reinforced product, and the second barrier is caused by a sprayed film.

If the shear modulus of the base crystal is less than the shear modulus of the deposited layer, the force acting on the side of this layer tends to push the dislocation away from the phase interface, which increases the strength of the product.

The higher the film modulus (i.e., the larger $K$ is), the smaller the number of dislocations in the cluste (accumulation) at the same applied voltage. Thus, a crystal covered with a rigid film will be plastically deformed less than a crystal without a film under the same stress (provided that the average path length of dislocations in the crystal is not very small). This may be the mechanism of primary hardening of crystals with hard coatings at an early stage of deformation. 


\section{References}

1. X.F. Zheng, F.X. Qin, H. Wang, Y.-W. Mai, H.X. Peng, Microwave 151, 62-70 (2017)

2. R. Li, N. Lachman, P. Florin, H.D. Wagner, B.L. Wardle, Composites Science and Technology 117, 139-145 (2015)

3. B. Yazdani, B. Chen, L. Benedetti, R. Davies, Y. Zhu, Composites Science and Technology 167, 243-250 (2017)

4. K. Brandt, V. Salikov, H. Özcoban, P. Staron, G.A. Schneider, Composites Science and Technology 72, 65-71 (2011)

5. W. Li, M. Chen, Z. Zeng, H. Jin, Z. Zhang, Composites Science and Technology 145, 10-14 (2017)

6. V.V. Ivanov, S.I. Popov, A.V. Kirichek, Key Engineering Materials 736, 18-22 (2017) doi:10.4028/ www.scientific.net/ KEM.736.18

7. V.V. Ivanov, N.S. Dontsov, A.V. Kirichek, Key Engineering Materials 736, 105-109 (2017) doi:10.4028/www.scientific.net/ KEM.736.105

8. Lei Guo, Zheng Yan, Xue Dong, Xichun Liu, Fuxing Ye, Composites Science and Technology 161, 473-482 (2019)

9. X.F. Zheng, F.X. Qin, H. Wang, Y.-W. Mai, H.X. Peng, Inter-fiber stresses in composites with carbon nanotube grafted and coated fibers 151, 62-70 (2017)

10. Sung-Jun Joo, Myeong-Hyeon Yu, Won Seock Kim, Hak-Sung Kim, Composites Science and Technology 167, 62-70 (2017)

11. M. Sharma, S. Gao, E. Mäder, H. Sharma, J. Bijwe, Composites Science and Technology 102, 35-50 (2014)

12. Bo Jiang, Tong Zhang, Liwei Zhao, Yudong Huang, Composites Science and Technology 140, 39-45 (2017)

13. V.V. Ivanov, S.I. Popov, A.V. Kirichek, IOP Conference Series: Materials Science and Engineering 327(3), 032026 (2018) doi:10.1088/1757-899X/327/3/032026

14. V.V. Ivanov, N.S. Dontsov, A.V. Kirichek, IOP Conference Series: Materials Science and Engineering 327(3), 032025 (2018) doi:10.1088/1757-899X/327/3/032025 